\title{
The Conservation of VIT1-Dependent Iron Distribution in Seeds
}

\author{
Seckin Eroglu ${ }^{1 *}$, Nur Karaca ${ }^{2}$, Katarina Vogel-Mikus ${ }^{3,4}$, Anja Kavčič $^{3}$, Ertugrul Filiz ${ }^{5}$ and \\ Bahattin Tanyolac ${ }^{2}$
}

'Department of Genetics and Bioengineering, Izmir University of Economics, Izmir, Turkey, ${ }^{2}$ Department of Bioengineering, Ege University, Izmir, Turkey, ${ }^{3}$ Department of Biology, University of Ljubljana, Ljubljana, Slovenia, ${ }^{4}$ Jozef Stefan Institute, Ljubljana, Slovenia, ${ }^{5}$ Department of Crop and Animal Production, Cilimli Vocational School, Duzce University, Duzce, Turkey

One third of people suffer from anemia, with iron (Fe) deficiency being the most common reason. The human diet includes seeds of staple crops, which contain Fe that is poorly bioavailable. One reason for low bioavailability is that these seeds store Fe in cellular compartments that also contain antinutrients, such as phytate. Thus, several studies have focused on decreasing phytate concentrations. In theory, as an alternative approach, Fe reserves might be directed to cellular compartments that are free of phytate, such as plastids. However, it is not known if seed plastid can represent a major Fe storage compartment in nature. To discover distinct types of Fe storage in nature, we investigated metal localizations in the seeds of more than

OPEN ACCESS

Edited by:

Thomas J. Buckhout, Humboldt University of Berlin,

Germany

Reviewed by:

Louis Grillet,

Academia Sinica, Taiwan

James Connorton John Innes Centre (JIC)

United Kingdom

*Correspondence:

Seckin Eroglu

erogluseckin@gmail.com

Specialty section:

This article was submitted to Plant Nutrition,

a section of the journal

Frontiers in Plant Science

Received: 19 December 2018

Accepted: 26 June 2019

Published: 12 July 2019

Citation:

Eroglu S, Karaca N,

Vogel-Mikus K, Kavčič A, Filiz E and Tanyolac B (2019) The Conservation of VIT1-Dependent Iron Distribution in Seeds. Front. Plant Sci. 10:907.

doi: 10.3389/fp/s.2019.00907 twenty species using histochemical or X-ray based techniques. Results showed that in Rosids, the largest clade of eudicots, Fe reserves were primarily confined to the embryo of the seeds. Furthermore, inside the embryos, Fe accumulated specifically in the endodermal cell layer, a well-known feature that is mediated by VACUOLAR IRON TRANSPORTER1 (VIT1) in model plant Arabidopsis thaliana. In rice, Fe enrichment is lost around the provasculature in the mutants of VIT1 orthologs. Finally, in Carica papaya, Fe accumulated in numerous organelles resembling plastids; however, these organelles accumulated reserve proteins but not ferritin, failing to prove to be plastids. By investigating Fe distribution in distinct plant lineages, this study failed to discover distinct Fe storage patterns that can be useful for biofortification. However, it revealed Fe enrichment is widely conserved in the endodermal cell layer in a VIT1-dependent manner in the plant kingdom.

Keywords: biofortification, seed, iron, metal, vit1, plastid, synchrotron, homeostasis

\section{INTRODUCTION}

One third of people suffer from anemia, with iron (Fe) deficiency being the most common reason (Kassebaum et al., 2014). Human diets are largely based on plants, which are often a poor source of $\mathrm{Fe}$ (Gibson et al., 2010). This is because corn, wheat, and rice kernels contain low concentrations of total Fe, and a large part of it cannot be absorbed by the human digestive system (Borg et al., 2009). To combat Fe deficiency, increasing bioavailable Fe (biofortification) of seeds has been suggested as the most sustainable approach (Shahzad et al., 2014).

Classical breeding, genetic engineering and other methods have been applied to increase $\mathrm{Fe}$ concentration in seeds (Murgia et al., 2012). Classical breeding has resulted in limited success, especially when natural cultivars show low variability in seed Fe levels. Genetic engineering 
methods have been shown to successfully increase Fe levels in major crops such as rice and wheat (Lee et al., 2009; Connorton et al., 2017). This increase has been mainly achieved by overexpressing proteins that boost the Fe storage capacity of the cells, eventually forcing the mother plant to divert more Fe into the seeds. Most of the biofortification approaches have targeted increasing concentration of Fe, but its low bioavailability is another major concern.

Bioavailability determines the portion of Fe that can be absorbed by the human digestive system. Fe absorption is affected by the form of $\mathrm{Fe}$, which may lead to less than ten percent of the total Fe intake (Hurrell and Egli, 2010). Staple crop species accumulate $\mathrm{Fe}$ in cells that contain metal binding molecules, with phytate being the most important. Fe releases from phytate due to the presence of phytases to support germination (Hegeman and Grabau, 2001). However, humans do not possess phytase, therefore phytates act as antinutrients to humans. To increase Fe bioavailability, several studies have aimed at decreasing phytate concentrations (Shi et al., 2007; Reddy et al., 2017). Such a decrease has been achieved but at the expense of growth (Warkentin et al., 2012). An alternative approach could be to redirect $\mathrm{Fe}$ from phytate-containing compartments to phytate-free compartments. In nature, at a subcellular level, Fe accumulates in vacuoles, plastids, cytosol or elsewhere (Otegui et al., 2002; Cvitanich et al., 2010). In vacuoles, Fe accumulates in globules where it binds to phytate (DavilaHicks et al., 2004; Lanquar et al., 2005). In plastids, Fe binds to ferritin protein (Waldo et al., 1995; Briat et al., 2010). In contrast to phytate-bound Fe, ferritin-bound $\mathrm{Fe}$ is bioavailable (Davila-Hicks et al., 2004). Thus, optimizing seed Fe for human nutrition requires not only increasing Fe concentration but also its bioavailability, which is strictly dependent on its subcellular level distribution.

The advances in Fe imaging techniques have allowed detailed investigations of Fe localization in the small seeds of Arabidopsis thaliana (Eroglu, 2018). The X-ray synchrotron fluorescence imaging technique identified nonhomogenous distribution of $\mathrm{Fe}$ throughout the embryo and revealed an Fe-enriched hotspot around the provascular strands (Kim et al., 2006). However, the emerging technique failed to pinpoint the specific cells that are overaccumulating Fe. Such a resolution has been achieved with the use of an enhanced version of classical Perls staining, namely, Perls/DAB (Roschzttardtz et al., 2009). Perls/DAB revealed that the Fe enrichment around provascular strands is confined to endodermal cells (Roschzttardtz et al., 2009; Ramos et al., 2013).

The genetic basis of endodermal Fe accumulation has been tackled by the comparison of T-DNA insertion mutants of A. thaliana. Two individual lines lost the endodermal Fe enrichment when mutated in the VACUOLAR IRON TRANSPORTER1 (VIT1) gene (Kim et al., 2006; Eroglu et al., 2017). VIT1 localized to the tonoplast, expressed in the stele, and complemented the Fe hypersensitivity of yeast when expressed heterologously; suggesting it imports $\mathrm{Fe}$ into the vacuoles of endodermal cells (Kim et al., 2006). Disruption of VIT1 relocalized Fe from the endodermal to the subepidermal cell layer in vit1 mutants. vit1 mutants further lost the subepidermal Fe enrichment when mutated in METAL TOLERANCE PROTEIN8 (MTP8) (Eroglu et al., 2016, 2017; Eroglu, 2018). Similar to VIT1, MTP8 localized to the tonoplast and complemented the Fe hypersensitivity of yeast (Eroglu et al., 2017). The disruption of VIT1 and MTP8 together relocalized Fe throughout the embryo homogeneously. In conclusion, analysis of loss-offunction mutants of $A$. thaliana shows that metal transporters determine where metals will be stored in the mature seed. This indicates that if a suitable Fe transporter protein is used, it might be possible to direct Fe to certain organelles that are free of antinutrients.

Our research aim was to identify a seed Fe transporter that can be useful to direct Fe to the plastids. A. thaliana accumulates Fe almost exclusively inside the vacuoles, therefore failing to be a suitable model for such an approach. Fe distribution in the seeds of other plants is poorly known. To find plastid targeting Fe transporters, we screened Fe distributions in seeds belonging to distinct plant lineages. Based on what is known from the model plant, we hypothesized that Fe concentrated regions will indicate the presence of a transporter. To this end, we examined the seeds of more than 20 different species belonging to several different plant orders by using Perls/DAB histochemical staining or synchrotron X-ray fluorescence spectroscopy.

\section{MATERIALS AND METHODS}

\section{Collection of Seeds}

Seeds were obtained from gene banks, local suppliers, or personally collected (Table 1 ). Rice T-DNA insertion mutants vit1-1 (Os04g0463400 Zhonghua11 background) and vit2-1 (Knock out line of Os09g0396900, Dongjin genetic background) and wild type (Dongjin) were courtesy of Dr. Ji-Ming Gong (Zhang et al., 2012).

\section{Perls Staining and $\mathrm{DAB} / \mathrm{H}_{2} \mathrm{O}_{2}$ Intensification (Perls/DAB)}

Wherever possible (embryos were large, could easily be isolated, seed coat was sufficiently soft to be cut, and high magnification was not needed), samples were directly (i.e., without fixation) stained by Perls (Figures 4A,B) or Perls/DAB (Figures 2C, 3D) (Roschzttardtz et al., 2009). In other cases, in situ protocol was used. For direct Perls staining, samples were vacuum infiltrated in $4 \%(\mathrm{v} / \mathrm{v}) \mathrm{HCl}$ and 4\% (w/v) K-ferrocyanide (Perls stain solution) for $15 \mathrm{~min}$ and incubated for $30 \mathrm{~min}$ at room temperature (Stacey et al., 2008). If the Fe signal was weak, it was intensified using DAB (Roschzttardtz et al., 2009). Perls-stained samples were incubated in a methanol solution containing $0.01 \mathrm{M} \mathrm{NaN}_{3}$ and $0.3 \%(\mathrm{v} / \mathrm{v}) \mathrm{H}_{2} \mathrm{O}_{2}$ for $1 \mathrm{~h}$, and then washed with $0.1 \mathrm{M}$ phosphate buffer ( $\mathrm{pH}$ 7.4). For the intensification reaction, samples were incubated between 10 and $30 \mathrm{~min}$ in a $0.1 \mathrm{M}$ phosphate buffer $(\mathrm{pH}$ 7.4) solution containing $0.025 \%(\mathrm{w} / \mathrm{v}) \mathrm{DAB}, 0.005 \%(\mathrm{v} / \mathrm{v}) \mathrm{H}_{2} \mathrm{O}_{2}$, and $0.005 \%(\mathrm{w} / \mathrm{v}) \mathrm{CoCl}_{2}$. The reaction was stopped by rinsing with distilled water.

For in situ Perls staining, seeds were first fixed in $10 \%$ formalin and then dehydrated in an ethanol series $(30 \%, 40 \%, \ldots$, $100 \%)$. Seeds were cleared with xylene. Seeds that were still 
TABLE 1 | Seeds used in the study.

\begin{tabular}{ll}
\hline Species & Source \\
\hline Linum usitatiss & National Genebank, Menemen, Turkey \\
Medicago truncatula & National Genebank, Menemen, Turkey \\
Cucumis sativus & National Genebank, Menemen, Turkey \\
Citrullus lanatus & National Genebank, Menemen, Turkey \\
Cannabis sativa & National Genebank, Menemen, Turkey \\
Euonymus europaeus & National Genebank, Menemen, Turkey \\
Alyssum sibiricum & National Genebank, Menemen, Turkey \\
Brassica oleracea & National Genebank, Menemen, Turkey \\
Gossypium arboreum & National Genebank, Menemen, Turkey \\
Batis maritima & Kew Gardens, London, England \\
Moringa peregrina & Kew Gardens, London, England \\
Eucalyptus elata & Kew Gardens, London, England \\
Geranium carolinianum & Kew Gardens, London, England \\
Brassica napus & Genebank, Gatersleben, Germany \\
Limnanthes douglasii & Botanischer Garten, Marburg, Germany \\
Arabidopsis thaliana & Arabidopsis Biological Resource Center, \\
Reseda lutea & Ohio, United States \\
Noccaea praecox & Personally collected* \\
Carica papaya & Personally collected** \\
Arachis hypogaea & Commercial \\
Solanum lycopersicum & Commercial \\
Capparis spinosa & Commercial \\
\hline
\end{tabular}

*Çilden et al., $2018{ }^{* *}$ Collected from Pb, Zn polluted site, Mežica, Slovenia Nogel-Mikus et al., 2005).

too hard to cut were further incubated in $20 \%$ formic acid for $45 \mathrm{~min}$. Seeds were then embedded in wax and 10-30 $\mu \mathrm{m}$ sections were cut. Sections were deparaffinized by incubation at $65^{\circ} \mathrm{C}$ for $15 \mathrm{~min}$ following a xylene treatment for $15 \mathrm{~min}$, and were stained with Perls/DAB according to Roschzttardtz et al. (2009). Sections were treated with $4 \%(\mathrm{v} / \mathrm{v}) \mathrm{HCl}$ and $4 \%(\mathrm{w} / \mathrm{v})$ K-ferrocyanide (Perls stain solution) for $15 \mathrm{~min}$ and incubated for $30 \mathrm{~min}$ at room temperature (Stacey et al., 2008). DAB intensification was applied as described in Meguro et al. (2007). For the intensification reaction, the sections were incubated between 10 and $30 \mathrm{~min}$ in a $0.1 \mathrm{M}$ phosphate buffer $(\mathrm{pH} 7.4)$ solution containing $0.025 \%(\mathrm{w} / \mathrm{v}) \mathrm{DAB}($ Sigma), $0.005 \%(\mathrm{v} / \mathrm{v})$ $\mathrm{H}_{2} \mathrm{O}_{2}$, and $0.005 \%(\mathrm{w} / \mathrm{v}) \mathrm{CoCl}_{2}$ (intensification solution). The reaction was stopped by rinsing samples with distilled water. Samples were imaged with a light microscope (Axioskop; Carl Zeiss, Jena, Germany).

For iodine staining, sections were stained with Gram's iodine solution ( $6.7 \mathrm{~g}$ potassium iodide and $3.3 \mathrm{~g}$ iodine dissolved in $1 \mathrm{~L}$ $\mathrm{H} 2 \mathrm{O}$; Ward's science) for at least $5 \mathrm{~s}$.

For Naphthol Blue-Black staining of proteins, slides were placed in $0.1 \%(\mathrm{w} / \mathrm{v})$ Naphthol Blue-Black, $10 \%(\mathrm{v} / \mathrm{v})$ acetic acid for $5 \mathrm{~min}$. For immunohistochemistry, after deparaffinization, tissues were rehydrated by ethanol series and hybridization was conducted according to Paciorek et al. (2006). Anti-rabbit secondary antibody was conjugated with Alexa-488 and was used with 1:800 dilution (Jackson ImmunoResearch, West Grow, PA, United States).

\section{X-Ray Fluorescence}

$\mathrm{X}$-ray fluorescence imaging of Brassica napus, Moringa peregrine, and Euonymus europaeus was performed at XRF beamline of Synchrotron Elettra (Karydas et al., 2018) on $60 \mu \mathrm{m}$ seed crosssections. The seeds were imbibed overnight at $4^{\circ} \mathrm{C}$, flash frozen in propane cooled with liquid nitrogen, embedded in tissue freezing medium (Leica, Germany), and cut using CM3060 Leica Cryostat (Vogel-Mikuš et al., 2014). The sections were sandwiched between two $2.5 \mu \mathrm{m}$ Mylar foil and scanned by $75 \times 75 \mu \mathrm{m}$ beam at an excitation energy of $10 \mathrm{KeV}$. Obtained XRF spectra were fitted by PyMCA software (Solé et al., 2007) and quantified (Kump and Vogel-Mikuš, 2018). Imaging of element distribution in Noccaea praecox was performed by micro-PIXE as described (Vogel-Mikus et al., 2007; Vogel-Mikuš et al., 2008).

\section{Bioinformatics Analyses}

VIT1 protein sequences were derived from the Phytozome database 12.1.6 version ${ }^{1}$ (Goodstein et al., 2011) for bioinformatics analyses. The following plants were included: A. thaliana (AT2G01770), Carica papaya (evm.model.supercontig_2.168), Cucumis sativus (Cucsa.174130.1, Cucsa.012090, and Cucsa.012100), Linum usitatiss (Lus10021809), Medicago truncatula (Medtr8g105790 and Medtr8g105810), Eucalyptus grandis (Eucgr.B02690, Eucgr.B02691, and Eucgr.G02084), B. napus (A0A078JDR3), Gossypium arboreum (A0A0B0MD68), Arachis duranensis (XP_020996537.1), Arachis ipaensis (XP_020977382.1), Brachypodium distachyon (Bradi4g29720 and Bradi5g12570), Brassica rapa (Brara.B03068 and Brara.F03456), Chlamydomonas reinhardtii (Cre02.g099500 and Cre02.g107550), Glycine max (Glyma.05G24060 and Glyma.08G047500), Gossypium raimondii (Gorai.013G029700), Zea mays (GRMZM2G074672 and GRMZM2G107306), Vitis vinifera (GSVIVG01011628001 and GSVIVG01011629001), Oryza sativa (LOC_Os04g38940 and LOC_Os09g23300), Phaseolus vulgaris (Phvul.002G322800 and Phvul.002G322900), Populus trichocarpa (Potri.010G104100 and Potri.010G104200), Physcomitrella patens (Pp3c2_34540), Prunus persica (Prupe.1G335200 and Prupe.1G335300), Sorghum bicolor (Sobic.002G194600 and Sobic.006G109000), and Solanum lycopersicum (Solyc04g008060). Protein domains were detected using Pfam 31.02 (Finn et al., 2015). Sequence length, molecular weight, and isoelectric point $(p I)$ were determined by using the ExPASy ProtParam tool $^{3}$ (Gasteiger et al., 2005). The transmembrane helices were predicted by using The HMMTOP transmembrane topology prediction server version $2.0^{4}$ (Tusnady and Simon, 2001). The phylogenetic tree of VIT1s was constructed with MEGA 7.0.2 software (Kumar et al., 2016) using maximum likelihood (ML) method with 1000 bootstraps. The evolutionary distances were computed using the Poisson correction method (Zuckerkandl and Pauling, 1965).

\footnotetext{
${ }^{1}$ https://phytozome.jgi.doe.gov/pz/portal.html\#

${ }^{2}$ https://pfam.xfam.org/

${ }^{3}$ https://web.expasy.org/protparam/

${ }^{4} \mathrm{http} / / /$ www.enzim.hu/hmmtop/
} 

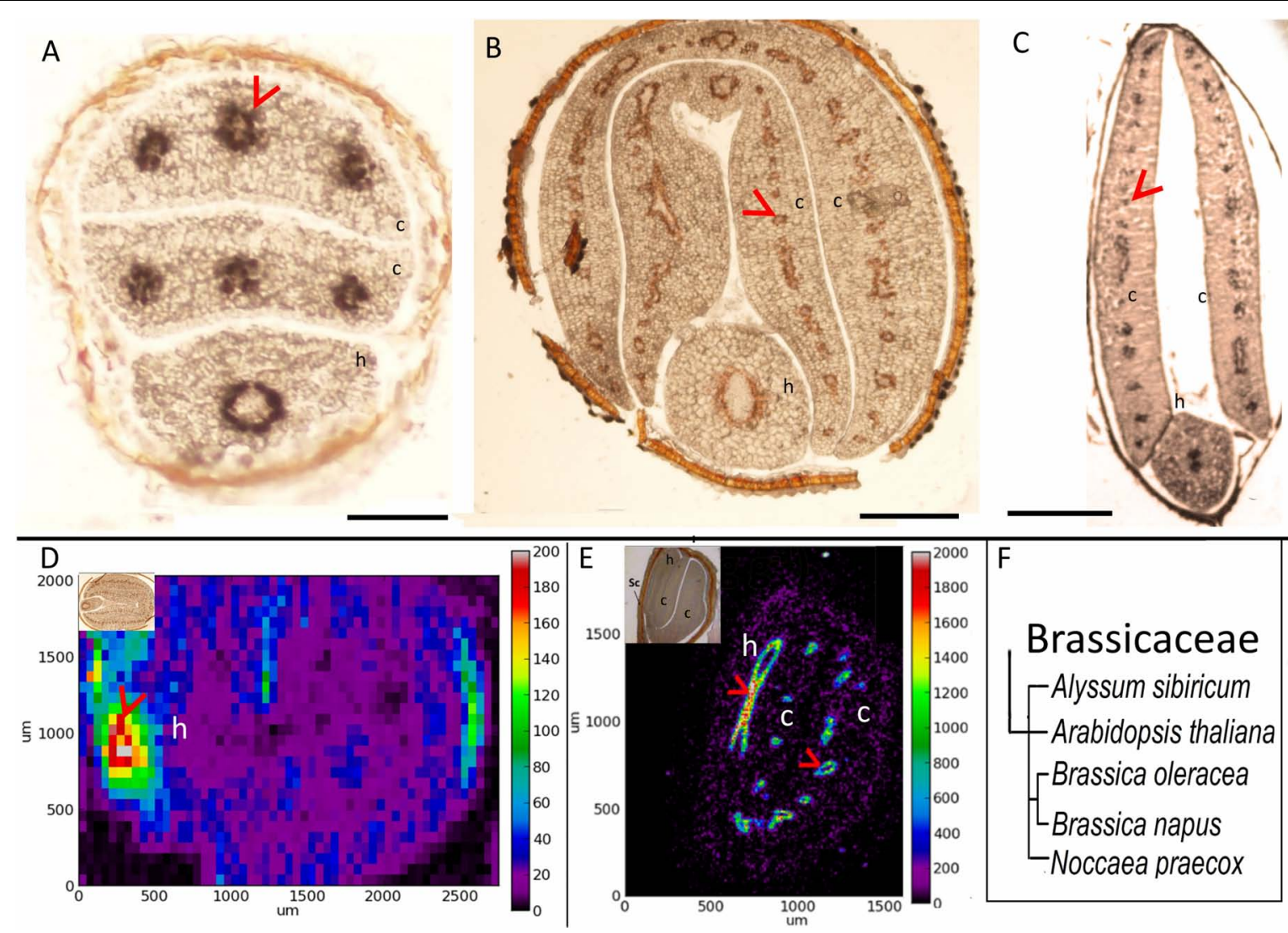

FIGURE 1 | Fe accumulates in the endodermis of Brassicaceae family. (A-C) Perls/DAB-stained seed cross sections. Cross sections were stained with Perls/DAB and observed under light microscope. From left to right: Arabidopsis thaliana, Brassica oleracea, and Alyssum sibiricum. Brown regions surrounding the embryos are seed coats. These were already brown before the staining; thus, the color does not reflect the stained Fe. Fe appeared as black stains in panels (A) and (C), and brown in panel (B). Circular-shaped staining in Arabidopsis thaliana corresponds to the endodermal cells surrounding the provascular strands. (D,E) Synchrotron X-Ray fluorescence images of relative Fe distribution in the seeds. (D) Brassica napus and (E) Noccaea praecox. (F) Branch of the taxonomic tree, for the whole tree refer to Figure 8. This branch shows species that were examined for Fe reserves in Brassicaceae family and used as a visual aid. Note that Arabidopsis thaliana, Alyssum sibiricum, and Noccaea praecox, consist of a single pair of cotyledons, but both Brassica napus and Brassica oleracea consist of a pair of inner and an outer cotyledons. Bar represents $0.1 \mathrm{~mm}$ in panel (A), $0.5 \mathrm{~mm}$ in panels (B,C). c, cotyledon; h, hypocotyl. Red arrow heads point to examples of specific Fe accumulation pattern, closed rings around provasculature of cotyledons (A-C,E), and hypocotyl (D).

Identity values (\%) of VIT1s were analyzed using the NCBI blastp tool. ${ }^{5}$

In silico expression data of soybean VIT1 were obtained using the Seed Gene Network Database (www.seedgenenetwork.com). Arabidopsis VIT1 gene number AT2G01770 was submitted. That submission returned soybean (G. max) GeneChip Expression Profile for Probe Set - Gma.6465.1.S1_a_at corresponding to Glyma01g40600. The signal data of individual experiments were obtained from the original file (Geo number: GSE46906).

\section{RESULTS}

\section{Fe Accumulates in Endodermis in Brassicaceae Family}

To investigate how $\mathrm{Fe}$ distribution differs between $A$. thaliana and other members of Brassicaceae, by using Perls/DAB method

${ }^{5}$ https://blast.ncbi.nlm.nih.gov/Blast.cgi?PAGE=Proteins and X-ray synchrotron analysis, we compared Fe distribution in several different Brassicaceae species. A. thaliana showed circular Fe-enriched regions in cotyledons and hypocotyl (Figure 1A), confirming previous reports (Kim et al., 2006; Eroglu, 2018). This region corresponds to the endodermis (Roschzttardtz et al., 2009). Similar to A. thaliana (Figure 1A), all other members of Brassicaceae showed circular Fe-enriched regions, both in cotyledons and the hypocotyl (Figures 1B-E). Fe rings seemingly overlap the region surrounding provascular bundles, most probably endodermis, based on extrapolation from A. thaliana. B. napus, and Brassica oleracea contained conduplicate cotyledons (i.e., inner and outer), both of which exhibited the same Fe distribution pattern (Figures 1B,D). Despite the similarities in these patterns, variations were also observed between species. In contrast to A. thaliana (Figure 1A), the Fe-enriched region of $B$. napus was not confined to a single cell layer in the hypocotyl (Figure 1C). Furthermore, Alyssum sibiricum showed two adjacent Fe-enriched circles instead of one in its hypocotyl (Figure 1D). Taken together, results showed that 

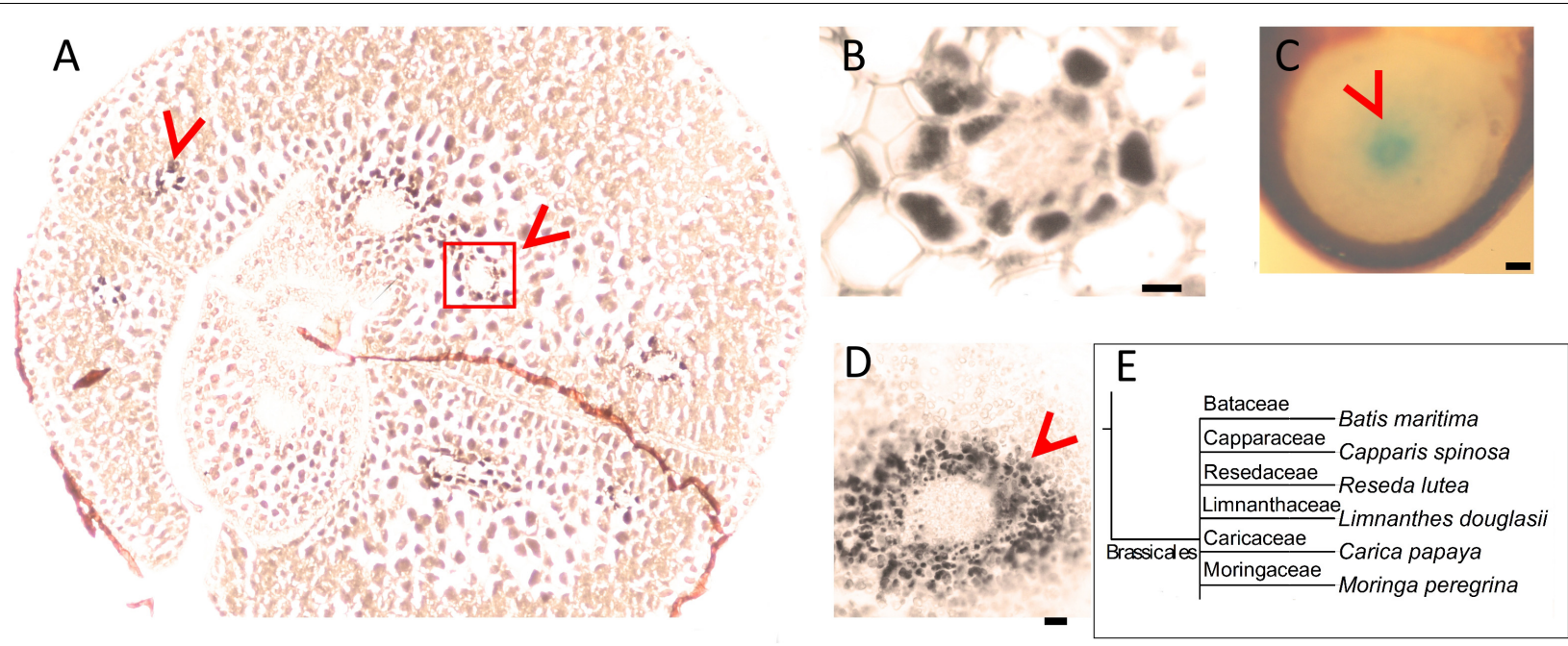

FIGURE 2 | Fe-enriched endodermis is conserved in Brassicales order. Seed cross sections were stained either with Perls or with Perls/DAB and observed under light microscope. (A) Limnanthes douglasii. (B) Higher magnification of the red square in panel (A). (C) Hypocotyl of Capparis spinosa. (D) Hypocotyl of Batis maritima. (B-D) Focusses on vascular tissues. Fe staining appeared black in panels (A,B,D) and blue in panel (C). Brown colored structure partially surrounding the embryo in panel (A) is the seed coat. This was already brown before the staining; thus, the color does not reflect the stained Fe. This branch shows species that were examined for Fe reserves in Brassicaceae family and used as a visual aid. Bar is $0.1 \mathrm{~mm}$ for panel (A,C); $0.01 \mathrm{~mm}$ for panel (B,D). (E) Branch of the taxonomic tree, for the whole tree refer to Figure 8. This branch shows species that were examined for Fe reserves in the same order but different families and used as a visual aid. Red arrow heads point to examples of specific Fe accumulation pattern, closed rings around provasculature of cotyledons $(\mathbf{A}, \mathbf{B})$ and hypocotyl $(\mathbf{C}, \mathbf{D})$.

not only A. thaliana, but also other Brassicaceae species store main Fe reserves in the endodermis.

\section{Conservation of Fe-Enriched Endodermis in the Order Brassicales}

Next, we assessed whether the conserved Fe pattern in A. thaliana seed extends beyond the family level to the order level. We searched for ring-like $\mathrm{Fe}$ distribution patterns around the provasculature of either the cotyledon or the hypocotyl, which was typical of Brassicaceae. We stained distinct species belonging to Brassicales by either Perls alone (for Fe-rich samples) or with DAB intensification (for Fe-poor samples) to reach a balance in staining intensity. In Limnanthes douglasii embryos, all cells showed the staining. At first glance, stained cells did not exclusively correspond to the endodermis (Figure 2A). However, closer examination revealed that cells around the provascular bundles of cotyledons were slightly enriched with Fe (Figure 2B). In Capparis spinosa, Perls staining without DAB amplification revealed that $\mathrm{Fe}$ accumulated close to the central cylinder of the hypocotyl (Figure 2C). In Batis maritima, Fe accumulated in several cell layers surrounding the provasculature, including the endodermis (Figure 2E). Taken together, an Fe-enriched endodermis is conserved in plants at least in order level.

\section{Fe Accumulation in the Endodermis Beyond the Order Brassicales}

Brassicales with 16 other orders together constitute a large clade of flowering plants, namely, Rosids (Chase et al., 2016). We next pursued the Fe distribution in species that belong to Rosids (Table 1). G. arboreum showed ring-like Fe distribution around provasculature (Figure 3A), similar to typical Festained endodermal cells. Furthermore, the rest of the cells were devoid of staining, indicating endodermis represented the main $\mathrm{Fe}$ reserves in the embryo. Likewise, Eucalyptus elata also showed $\mathrm{Fe}$ enrichment in the endodermis of the cotyledon. However, that of hypocotyl did not show the same enrichment (Figure 3B). We examined two species that belong to the Fagales order, namely, M. truncatula and Arachis hypogaea (Figures 3C,D). M. truncatula showed circular Fe-stained regions in the cotyledons, which represented the largest $\mathrm{Fe}$ pool in the embryo (Figure 3C). In contrast to $M$. truncatula, Fe seemingly accumulated homogeneously in A. hypogaea However, closer examination of A. hypogaea revealed provascular strands were enriched in $\mathrm{Fe}$ (Figure 3D).

\section{Conservation of VIT1 Sequence in Species That Do Not Show an Fe-Enriched Endodermis}

In $A$. thaliana seed, $\mathrm{Fe}$ enrichment in the endodermis is dependent on a functional VIT1 protein (Kim et al., 2006). We failed to observe an $\mathrm{Fe}$-enriched endodermis in some species. To evaluate how conserved VIT1 is in these species, VIT1 protein sequences in distinct plants were compared (Figure 4). First, to get a general picture, 34 sequences were determined and compared from 18 plant species (Figure 4A). In contrast to A. thaliana, which had only one VIT1, most plants showed two copies. Interestingly, in V. vinifera, one of the two copies of VIT1 clustered with monocot VITs in the phylogenetic tree. E. grandis contained three homologs of VIT1, one of which was distinctly 

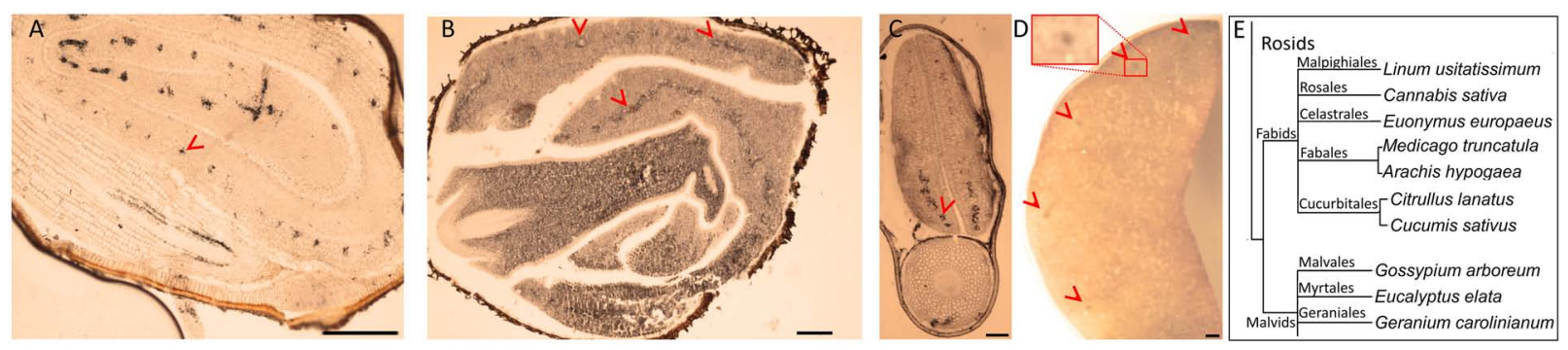

FIGURE 3 | Fe-enriched endodermis is conserved in distinct plant orders. Seed cross sections belonging to members of various orders were stained with Perls/DAB and observed under light microscope. From panels (A-C), Gossypium arboreum. Eucalyptus elata and M. truncatula, respectively. (D) Single cotyledon of Arachis hypogaea. Fe staining appeared black in panels (A-C) and brown in panel (D). Outer brown-black cover in panels (A-C) are seed coats. These were already brown before the staining, thus the color do not reflect the stained Fe. In panel (A), longitudinal section of hypocotyl and cross section of folded cotyledons can be differentiated. Red arrow heads show Fe enrichment surrounding the provascular strands of cotyledons. (E) Branch of the taxonomic tree, for the whole tree refer to Figure 8. This branch shows species that were examined for Fe reserves in distinct orders and used as a visual aid. Note that only selected examples from panel (E) is shown through (A-D). Bar is $1 \mathrm{~mm}$ for panel (A) and $0.1 \mathrm{~mm}$ for panels (B-D). Red arrow heads point to examples of specific Fe accumulation pattern, closed rings around provasculature of cotyledons.

diverged. These data suggest that VIT1 is well-conserved in distinct plant lineages (Supplementary Figures 1, 2).

We next assessed the conservation of VIT1 protein sequence in species that were used in $\mathrm{Fe}$ staining. The whole genome sequence was available only for A. thaliana, C. papaya, Cucumis sativus, L. usitatiss, M. truncatula, E. grandis, B. napus, G. arboreum, and Arachis sp. (Supplementary Figure 3). VIT1 sequences of all ten species consisted of 245-265 amino acids with five transmembrane helices and clustered in three subgroups (Supplementary Table 1). All contained the VIT1 domain structure (PF01988) (Supplementary Figure 2). Protein BLAST analyses showed that identity values (\%) of Arabidopsis and the other nine VIT1s ranged from 29 to $92 \%$ (Supplementary Figure 3). We found the highest identity value between Arabidopsis and B. napus (92\%), followed by M. truncatula and Linum usitasiss (83\%), while the lowest was found between A. thaliana and Arachis spp. (29\%), indicating variations of VIT1 genes in plants. All three VIT1 subgroups included members that stored Fe around the provasculature. Therefore, although some species did not exhibit a VIT1-dependent Fe enrichment in the endodermis (Supplementary Figure 4), their VIT1 sequences did not diverge from others.

In A. thaliana, analysis of ProVIT1::GUS lines showed VIT1 is preferentially expressed in the provasculature (Kim et al., 2006; Eroglu et al., 2017). To investigate whether spatial VIT1 expression is conserved in VIT1 orthologs, we compared expression of VIT1 orthologs using publicly available microarray databases. We found that VIT1 signal was highest in the provasculature in soybean embryo (Figure 4B). This data indicated spatial expression pattern is conserved in VIT1 orthologs.

Furthermore, to investigate whether VIT1's function is also conserved, we compared Fe distributions in mutants of VIT1 orthologs. VIT1 orthologs have been reported to involve in Fe distribution in rice embryo (Zhang et al., 2012; Bashir et al., 2013); however, precise description of the effect was lacking due to the low resolution, where Perls staining was applied without DAB amplification. Perls/DAB staining revealed that
Fe specifically concentrated around the provasculature in wild type rice and not in single vit1 nor vit2 mutants (Figure 4C). $\mathrm{Fe}$ accumulated less heterogeneously among embryonic tissues in those compared to the wild type. This data suggested $\mathrm{Fe}$ enrichment around provasculature is conserved and mediated by VIT1 homologs in rice.

\section{Organ, Tissue, and Subcellular Level Variations in Seed Fe Storage}

Perls staining revealed $\mathrm{Fe}$ storing protein bodies show great variation in the structure. C. papaya seed showed intense staining in the center corresponding to the embryo - even though the DAB intensification step was skipped -; while the peripheries were devoid of staining, corresponding to the endosperm (Figures 5A,B). At the tissue and subcellular level, staining was confined to organelles similar to leucoplasts regarding the shape, size, and number (Figure 5C). Distribution of this organelle did not differ between the endodermis and other cell layers. Since a specific group of seed leucoplasts store starch (i.e., amyloplast), we next investigated whether $\mathrm{Fe}$-accumulating organelles belong to that group. Iodine and periodic acid could only weakly stained those (data not shown), failing to show these organelles accumulate starch. Next, since Fe in plastids is associated with ferritin, using immunohistochemistry, we investigated if $\mathrm{Fe}$ accumulating organelle contains ferritin. Ferritin fluorescence were observed in the embryo cells but outside of those organelles (Figure 5D). Instead, unlike plastids, these organelles accumulated proteins, as shown by Naphthol Blue-Black staining (Figure 5E). Taken together, these data suggested accumulating organelles in C. papaya were indeed protein storage vacuoles or globules similar to many other plant seeds, despite their distinct relative size and appearance.

Nutrients accumulate usually in embryo part of the seeds. We found that in addition to the embryo, the endosperm and even the seed coat can significantly store $\mathrm{Fe}$ in a few Rosid species. For example, E. europaeus contained a large endosperm, which was intensely stained by Perls/DAB (Figures 6A,B). 
A

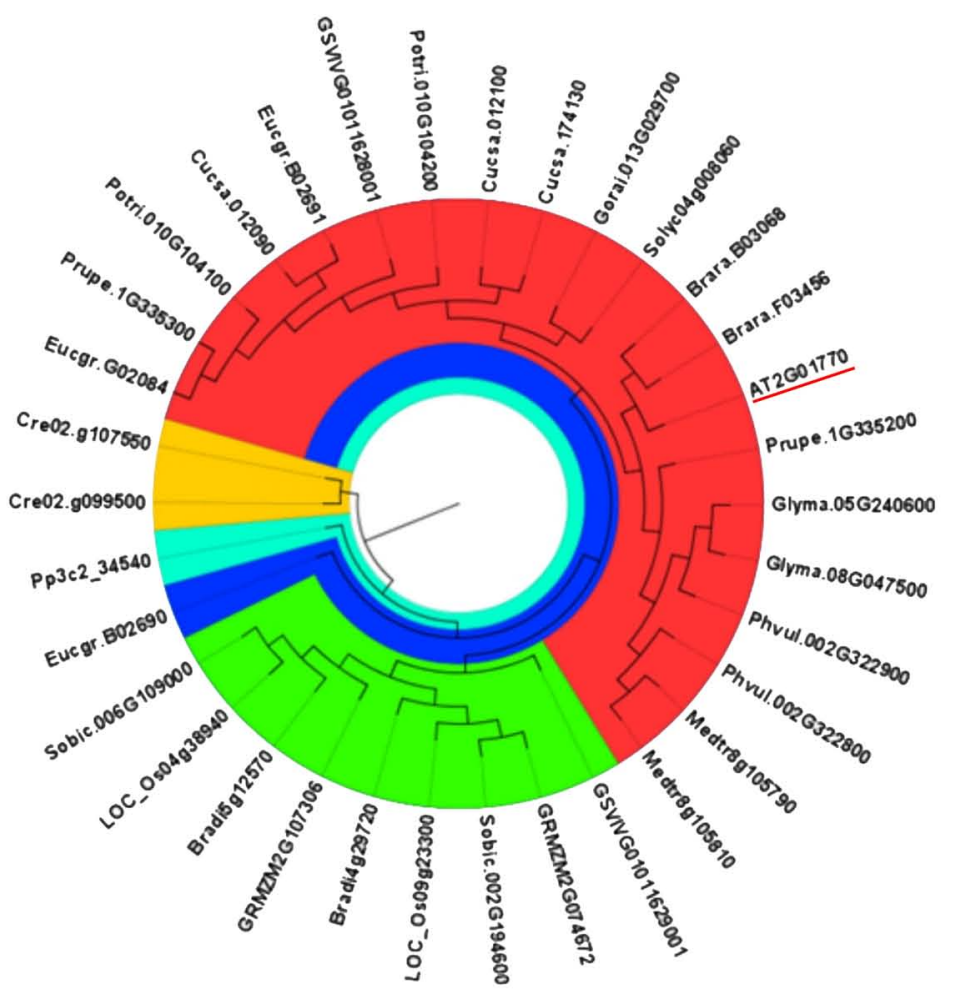

B

Expression of VIT1 ortholog in soybean embryo
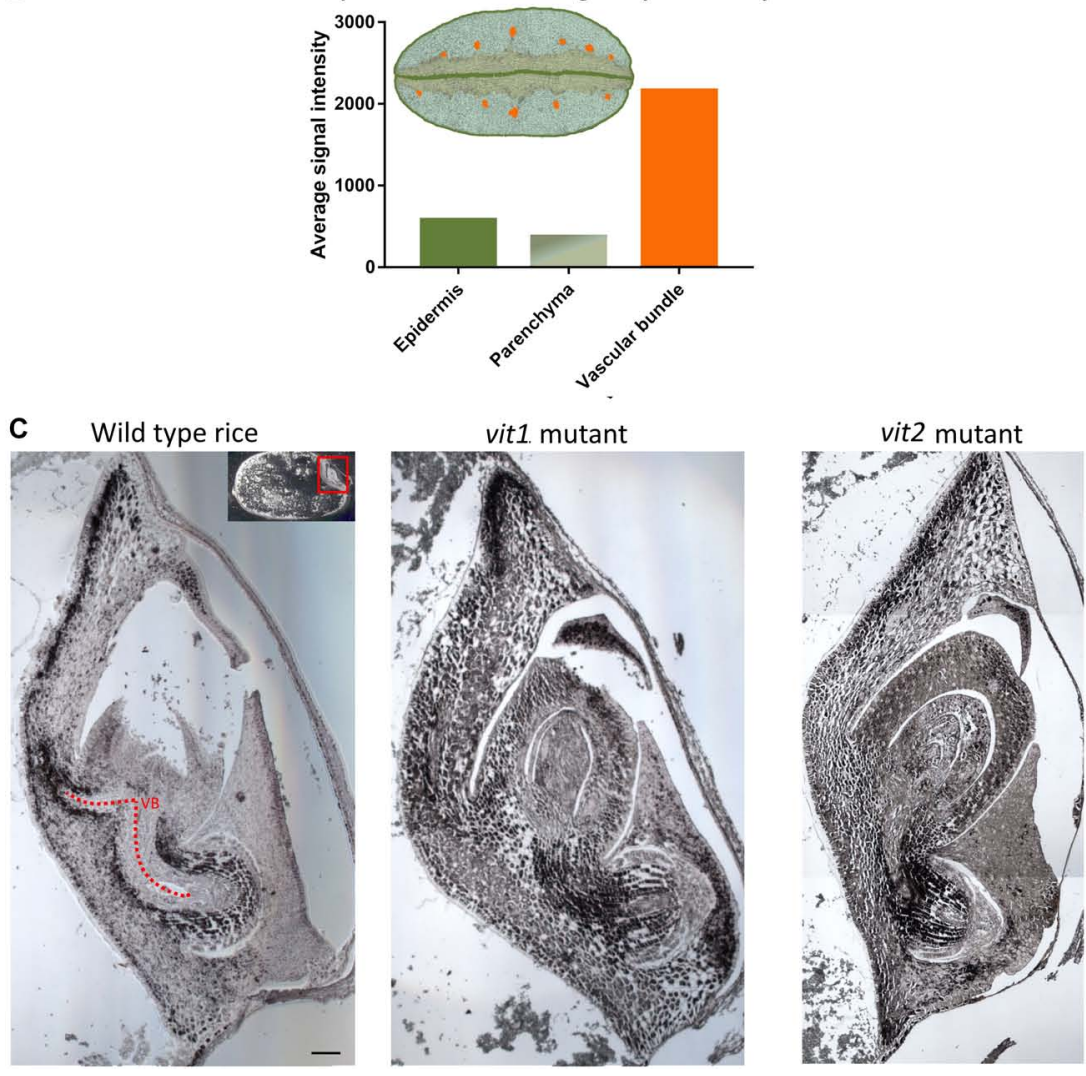

FIGURE 4 | Continued 
FIGURE 4 | Conservation of VIT1 sequence, expression and function. (A) Phylogenetic distribution of VIT1 orthologs in 18 different plant species from monocots, dicots and lower plants. Phylogeny was constructed by MEGA 7 with ML method for 1000 bootstraps using putative 34 VIT1 protein sequences. The red segment includes only dicots. Green segment includes only monocots except for one member of Vitis vinifera. The blue segment shows a diverged dicot member of E. grandis. Orange and cyan colors show algal and moss species, respectively. VIT1 from Arabidopsis thaliana is underlined. For details on analyzed sequences, refer to materials and method section. (B) Expression of VIT1 ortholog (Glyma01g40600) in embryo tissues of soybean (Microarray data retrieved from www.seedgenenetwork.net). The picture illustrates the soybean (Glycine max) tissues: green, epidermis; orange vascular bundles; rest, parenchyma. (C) Impact of VIT1 orthologs in Fe distribution of rice (Oryza sativa) embryo. Unlike dicots, monocots such as rice do not have well defined organs. Longitudinal sections of whole rice embryos were obtained from either wild type or mutant rice (VIT1 or VIT2 genes were either substantially knocked down or knocked out), stained by Perls/DAB (black color) and Fe distribution in the embryos were compared. Region with red line corresponds to provascular bundles (VB) in rice embryo. Red arrow heads point specific Fe accumulation pattern around provasculature. Bar is $0.1 \mathrm{~mm}$.
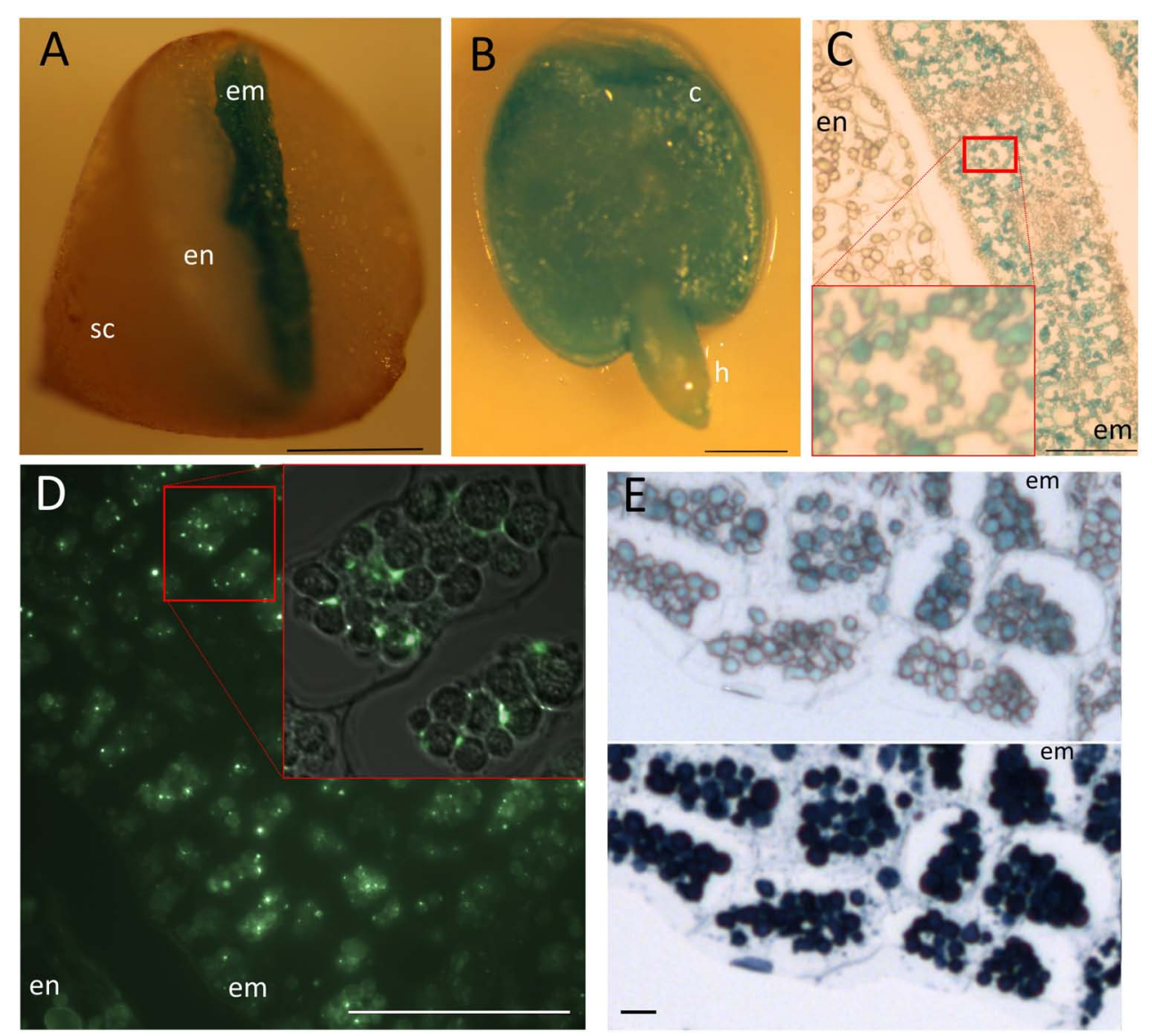

FIGURE 5 | Fe storage in Carica papaya. Distribution of Fe as subcellular hotspots in Carica papaya seeds. Carica papaya seeds were cleaned from the pericarp. (A) Seeds were cut into half by hand and were stained by Perls. (B) Whole embryo was isolated from the seed and was stained by perls. (C) Perls-stained thin cross section of a cotyledon. Red squares indicate close up. (D) Immunohistochemistry using ferritin antibodies. Bright green spots indicate ferritin presence. Red square indicates closeup, fluorescence image overlayed on top of the light microscopy image to indicate localization of fluorescent spots with regards to the Fe storing organelles. (E) Juxtaposition of Fe-stained and protein-stained regions of the embryo cross sections. The same cross section was first stained by Perls, pictured (top panel) and then stained by Naphthol Blue-Black, pictured again (bottom panel). Blue color in panel (A-C) and in the top panel of (E) indicate stained Fe. Note, the cellular position and number of the organelles slightly differ in panel (C), compared with (D) and (E). The difference might be due to a fixation artifact or cross sectioning from distinct regions of the embryos. Dark blue color in the bottom panel of (E) indicates presence of proteins. sc, seed coat; en, endosperm; C, cotyledon; h, hypocotyl; em, embryo. Bar is $1 \mathrm{~mm}$ for panels (A,B); $0.05 \mathrm{~mm}$ for panel (D); $0.1 \mathrm{~mm}$ for panel (C); $0.01 \mathrm{~mm}$ for panel (E).

As another example, the seed coat of Moringa peregrina was stained by Perls/DAB (Figures 6C,D). Interestingly, the staining intensity was higher in its seed coat compared with its embryo (Figure 6C), indicating that the seed coat is a major $\mathrm{Fe}$ accumulation site in M. peregrina.

Finally, the distinct distribution of Fe in E. europaeus and M. peregrina seeds was further investigated by synchrotron X-ray fluorescence spectrometry. Synchrotron X-ray fluorescence confirmed the Fe accumulation in the endosperm of E. europaeus and in the inner seed coat of $M$. peregrina (Figure 7). In addition to $\mathrm{Fe}$, synchrotron analysis further revealed the distribution of other metals. In E. europaeus, phosphorus (P) and Mn localized homogeneously through the embryo and endosperm, while zinc ( $\mathrm{Zn})$ localized exclusively in the embryo. In M. peregrina, $\mathrm{Fe}$ 

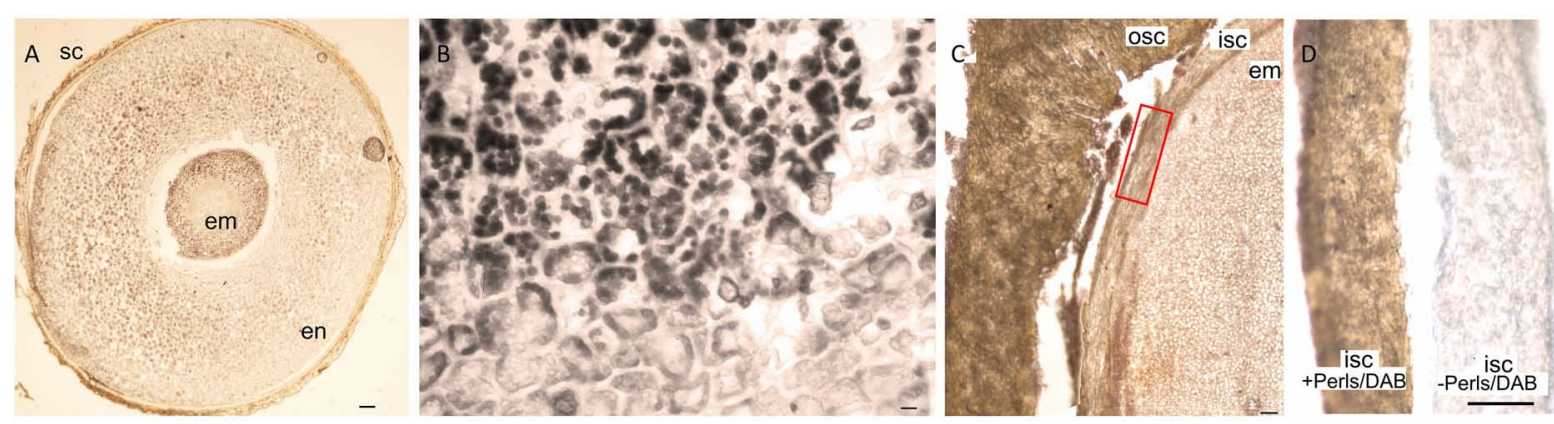

FIGURE 6 | Unusual Fe storage in the endosperm and in the seed coat. Fe staining in Euonymus europaeus and Moringa peregrina. Cross sections were stained with Perls/DAB and observed under light microscope. (A) Euonymus europaeus. (B) A close-up to endosperm tissue of Euonymus europaeus. (C) Moringa peregrina, focused on a small part of the seed. Embryo, inner and outer sections of the seed coats are visible. Moringa peregrina does not possess an endosperm and has a thick two-layered seed coat. (D) Dissected inner seed coats of Moringa peregrina. Left, Perls/DAB stained, right unstained control. Fe staining appeared brown in panels (A,C,D) and black in panel (B). Seed coat in panel (A) and outer seed coat in panel (C) were already brown before the staining, thus the color does not reflect the stained Fe. In contrast, inner seed coat of Moringa peregrina is white, and brown color in panel (C) shows the stained Fe. SC, seed coat; en, endosperm; osc, outer seed coat; isc, inner seed coat; em, embryo. Bar is $0.1 \mathrm{~mm}$ for panel (A), $0.01 \mathrm{~mm}$ for panel (B), $1 \mathrm{~mm}$ for panels (C,D).
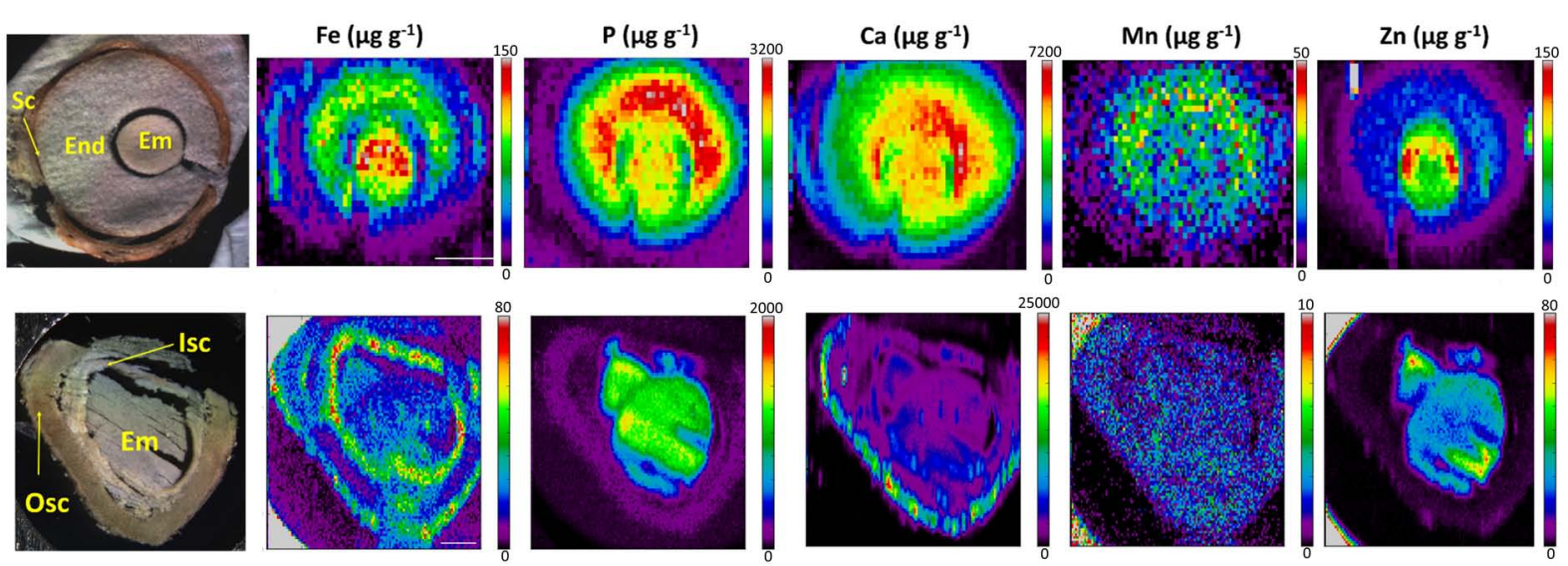

FIGURE 7|X-ray fluorescence spectroscopy confirms endosperm and seed coat accumulate Fe. Spatial distribution of metals in Euonymus europaeus (top panel) and Moringa peregrina (bottom panel). The first column of images shows light microscopy images. Other columns show distribution of elements; Fe, $\mathrm{P}, \mathrm{Ca}, \mathrm{Mn}$, and Zn, respectively. Colorbar indicates concentration. sc, seed coat; en, endosperm; isc, inner seed coat; osc, outer seed coat; em, embryo. Bar is 1 mm.

concentration was high not only in the inner seed coat as previously revealed by Perls/DAB (Figure 6) but also in the outer seed coat (Figure 7). Fe localized specifically to the outer side of the outer seed coat and throughout the inner seed coat. The outer seed coat of $M$. peregrina accumulated calcium (Ca), spatially overlapping Fe, but not other metals. Zn accumulated exclusively in the embryo.

\section{DISCUSSION}

We screened seeds that belong to distinct plant lineages using Perls/DAB staining and X-ray-based methods to find Fe-accumulating plastids. Although we failed to identify such seeds, our approach revealed seed Fe storage patterns in distinct plant lineages. Among those, Fe enrichment around provasculature is well conserved in Rosids which is dependent on orthologs of VIT1.
Fe accumulates almost exclusively in the embryos in Rosids (Figures 1-3, 5 and in all other plants that were examined in Figure 8). In contrast, monocots store large quantities of Fe also in the endosperm, specifically in the aleurone layer ( $\mathrm{Lu}$ et al., 2013; Singh et al., 2013, 2014; Vatansever et al., 2017). This organ level difference in Fe partition may be explained by the decrease in endosperm size during evolution (Forbis et al., 2002; FinchSavage and Leubner-Metzger, 2006). Branches that appear early, such as monocots, almost never possess an embryo occupying more than half of the total seed volume. In contrast, branches that appeared later (e.g., Rosids) can possess embryo that may fill almost the entire seed. In the latter, the endosperm shrinks as the seed develops and the embryo eventually occupies most of the seed volume. Since the endosperm is a nutritive tissue, as it degrades, nutrient storage function must be taken over by the embryo itself. Therefore, we suggest that the metal accumulation in the endosperm, at least for Fe, is taken over by the embryo as an evolutionary trend. 


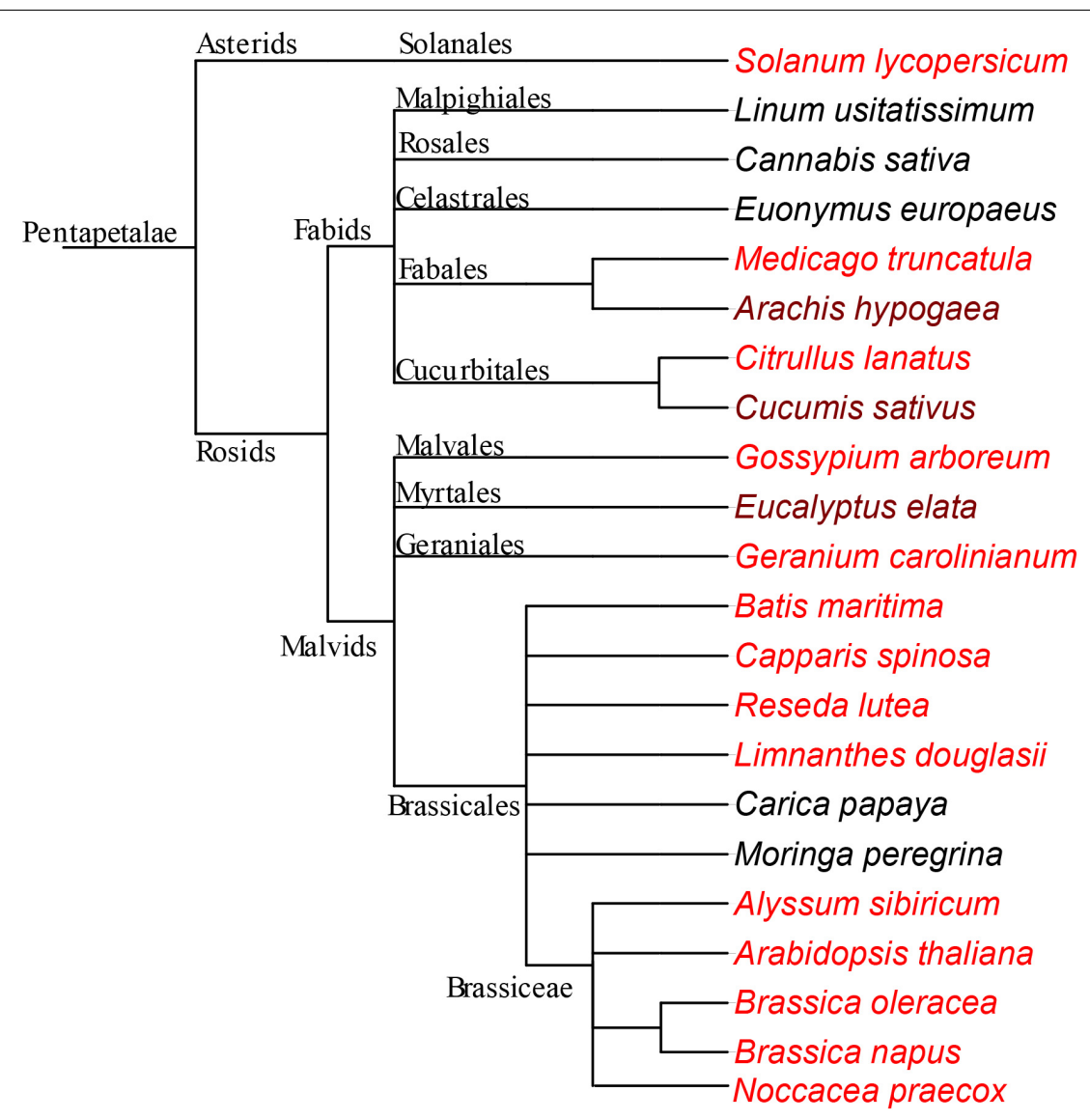

FIGURE 8 | Fe-enriched endodermis is a conserved feature in Rosids. Schema representing species that are used in this study. The phylogenetic tree was generated using NCBI taxonomy database and exported with the version 3 of the Interactive Tree of Life (iTOL) (Letunic and Bork, 2016). Both dark and light red colored species were enriched with Fe around provascular strands. In the darker-red-colored species, endodermis Fe does not represent the highest concentration of Fe, in contrast to the lighter-red-colored species. The species which are not red, do not specifically accumulate large Fe reserves in their endodermis. See discussion for the interpretation of the negative data.

Besides the general trends, Fe may accumulate in distinct organs and cellular compartments. The destination of nutrients must be the embryo, as the mature seed coat is dead while the endosperm is sterile. However, Fe accumulates in the seed coat higher than the embryo in rare cases (Figures 6, 7; Cvitanich et al., 2010; Moraghan et al., 2002). This can be related to the presence of chelators in the seed coat, such as tannins, which immobilize metal nutrients (Lombardi-Boccia et al., 1995). The function of trapped $\mathrm{Fe}$ reserves in the seed coat should be addressed in the future. In this regard, since most seed coats are naturally colored; Perls staining may fail to detect Fe; however, this problem can be circumvented by using fluorescent dyes (Park et al., 2014). In contrast to $\mathrm{Fe}, \mathrm{Zn}$ is not trapped in the seed coat (Figure 7). This might be due to its lower affinity to tannins and other polyphenols (Santos-Buelga and Scalbert, 2000). In Rosids, the endosperm rarely stores a significant amount of $\mathrm{Fe}$ (only in a few species, see Figures 6A,B, 7 and Supplementary Figure 4). Interestingly, $\mathrm{X}$-ray fluorescence showed $\mathrm{Fe}$ in the endosperm colocalizes with phosphorus (P) (Figure 7A). Localization of $\mathrm{P}$ usually mirrors phytate distribution in seeds, indicating $\mathrm{Fe}$ might be trapped by phytate in the endosperm before it can reach the embryo (Iwai et al., 2012). In summary, the principal storage organ for Fe in Rosid seeds is the embryo; however, Fe reserves can be restrained on the way to the embryo, most likely due to the immobilization by chelation in the endosperm or in the seed coat.

Carica papaya showed the most concentrated Fe hotspots among all the analyzed seeds. Despite the presence of a large endosperm, the Fe signal from Perls staining appeared exclusively in the embryo (Figure 5A), specifically localized in homogeneously distributed organelles similar to plastids (Figure 5B). To the best of our knowledge, plastids have never been reported as representing the main $\mathrm{Fe}$ reserves in any seeds. For example, Cvitanich et al. (2010) determined a large number of amyloplasts in beans, which even contain Fe-binding ferritin proteins; but these plastids were devoid of Perls stain. In papaya, we found that $\mathrm{Fe}$ accumulating organelles are related to protein storage vacuoles based on the absence of ferritin (Figure 5D) and presence of proteins (Figure 5E). Protein storage vacuole is the only vacuole plant seeds has and contain phytate enriched globules, where Fe reserves reside (Lanquar et al., 2005; Regvar et al., 2011). These globules are separated from each 
other, but often detected as a single aggregate under microscope (Roschzttardtz et al., 2009; Eroglu et al., 2017) which might be an artifact due to fixation (Herman and Larkins, 1999). Interestingly, in papaya, proteins aggregated in separated compartments (Figure 5). These compartments seemed to localize in the cytosol instead of being enclosed by a protein storage vacuole.

Endodermis represents the major conserved $\mathrm{Fe}$ hotspot in distinct plant lineages (Figure 8). In few species, Fe-enriched cells go beyond the single endodermal cell layer to nearby cortex cells [compare Figure 2B with 2D, also see Ibeas et al. (2017)]. Therefore, the question arises whether VIT1 can also localize to cortex cells in addition to the endodermis. VIT1 is able to localize to the inner-most cortex cells in the absence of the endodermis (Roschzttardtz et al., 2009), which may indicate - regardless of whether it is restricted to a single cell layer or not - that the typical ring-shaped Fe localization close proximity to provascular strands is due to VIT1. VIT1 plasticity may not be limited to the different number of cell layers and may extent to different subcellular localization. In beans, instead of the vacuole, Fe accumulated in the cytosol of endodermal cells (Cvitanich et al., 2010), indicating VIT1 orthologs can localize to the plasma membrane (as opposed to tonoplast in Arabidopsis). Taken together, these variations indicate the core signature of VIT1-mediated Fe accumulation in plants is the enrichment around the provasculature, not the confinement in the single cell layer nor the compartmentalization into the vacuole.

Although VIT1-mediated Fe enrichment was conserved, few species lacked this phenotype (Figures 5-8). This may raise the question of whether VIT1 has been lost in these species during the course of evolution. However, this is unlikely due to conservation of a VIT1 domain in species which lack a Fe-enriched endodermis (PF01988) (Figure 4A and Supplementary Figures 2, 3). Alternatively, we hypothesize that other transporters might have taken over VIT1's function in these species. Studies with loss of function mutants indicate that $\mathrm{Fe}$ patterns are eventually determined by a single dominant transporter (Kim et al., 2006; Eroglu et al., 2017). For example, in A. thaliana, VIT1's presence prevents another protein, MTP8, from storing Fe. Likewise, when a more preferential $\mathrm{Fe}$ transporter is present, VIT1 may still mediate $\mathrm{Fe}$ accumulation (i.e., Fe-enriched endodermis) but to a much lesser extent. Furthermore, the nature of techniques used in the study is biased in revealing a less pronounced Fe store in the presence of a highly pronounced one, making it likely to miss VIT1's impact in the presence of a stronger metal transporter. For instance, X-ray analysis (Kim et al., 2006) or Perls/DAB staining (Figure 1A) fail to detect any $\mathrm{Fe}$ in cortex or epidermal cells in the presence of the large endodermal Fe pool, although they constitute half of the total seed Fe (Ramos et al., 2013). Taken together, VIT1 and its associated phenotype is well-conserved despite some seeds showing alternative Fe hotspots.

\section{REFERENCES}

Bashir, K., Takahashi, R., Akhtar, S., Ishimaru, Y., Nakanishi, H., and Nishizawa, N. K. (2013). The knockdown of OsVIT2 and MIT affects iron localization in rice seed. Rice 6:31. doi: 10.1186/1939-8433-6-31
Rosids is a huge lineage including 80,000 species belonging to 147 families, comprising more than a third of all angiosperms (Soltis, 2005; Hedges and Kumar, 2009). The current study shows Rosid seeds store $\mathrm{Fe}$ in the embryo. This $\mathrm{Fe}$ is not equally distributed but most often concentrated in the innermost cell layers, the endodermis and sometimes the cortex, in a VIT1-dependent manner. This phenotype goes beyond dicots and may extend to monocots as shown in rice. Future studies should examine other clades beyond Rosids to pinpoint at which stage of plant evolution the Fe-enriched endodermis appeared as a new feature.

\section{AUTHOR CONTRIBUTIONS}

SE and NK collected the seeds and performed the histochemistry. $\mathrm{KV}-\mathrm{M}$ and AK carried out the X-ray analyses. EF performed the bioinformatics. SE and BT conceived and planned the project. SE wrote the manuscript with the contributions of all the authors.

\section{FUNDING}

This study was partially supported by the ARRS (Slovenian Research Agency) (P1-0212, J7-9418, and J7-9398) and internal fundings. The research leading to this result has been supported by the project CALIPSOplus under Grant Agreement 730872 from the EU Framework Programme for Research and Innovation HORIZON 2020.

\section{ACKNOWLEDGMENTS}

Thanks to Asci Murat Mihladiz (Capparis Research \& Development Center, Burdur, Turkey) and Dr. Emre Cilden (Hacettepe University, Ankara, Turkey) for sharing seed stocks with us. We gratefully acknowledge Dr. Ali Veral and his team, especially Ebru Çanlı (Ege University Hospital, Izmir, Turkey) for excellent technical help in preparing the histological sections. Thanks to Recep Vatansever and Ferhat Celep for fruitful discussions. We also acknowledge Primoz Pelicon and Primoz Vavpetič (JSI) for their help with PIXE analysis and Iva Bozicevic Mihalic (Elettra Sincrotrone Trieste) for her help with the synchrotron measurements. Elettra was acknowledged for the provision of the beamtime (Project 20175078).

\section{SUPPLEMENTARY MATERIAL}

The Supplementary Material for this article can be found online at: https://www.frontiersin.org/articles/10.3389/fpls.2019.00907/ full\#supplementary-material

Borg, S., Brinch-Pedersen, H., Tauris, B., and Holm, P. B. (2009). Iron transport, deposition and bioavailability in the wheat and barley grain. Plant Soil 325, 15-24. doi: 10.1007/s11104-009-0046-6

Briat, J.-F., Duc, C., Ravet, K., and Gaymard, F. (2010). Ferritins and iron storage in plants. Biochim. Biophys. Acta 
BBA Gen. Subj. 1800, 806-814. doi: 10.1016/j.bbagen.2009. 12.003

Chase, M. W., Christenhusz, M. J. M., Fay, M. F., Byng, J. W., Judd, W. S., Soltis, D. E., et al. (2016). An update of the angiosperm phylogeny group classification for the orders and families of flowering plants: APG IV. Bot. J. Linn. Soc. 181, 1-20. doi: 10.1111/boj.12385

Çilden, E., Yildirimli, Ş, Zare, G., and Martín-Bravo, S. (2018). Rediscovery of the restricted endemic Reseda balansae (Resedaceae) in Turkey. Phytotaxa 362, $87-96$.

Connorton, J. M., Jones, E. R., Rodríguez-Ramiro, I., Fairweather-Tait, S., Uauy, C., and Balk, J. (2017). Wheat vacuolar iron transporter TaVIT2 Transports Fe and Mn and Is effective for biofortification. Plant Physiol. 174, 2434-2444. doi: 10.1104/pp.17.00672

Cvitanich, C., Przybyłowicz, W. J., Urbanski, D. F., Jurkiewicz, A. M., MesjaszPrzybyłowicz, J., Blair, M. W., et al. (2010). Iron and ferritin accumulate in separate cellular locations in Phaseolus seeds. BMC Plant Biol. 10:26. doi: 10.1186/1471-2229-10-26

Davila-Hicks, P., Theil, E. C., and Lönnerdal, B. (2004). Iron in ferritin or in salts (ferrous sulfate) is equally bioavailable in nonanemic women. Am. J. Clin. Nutr. 80, 936-940. doi: 10.1093/ajcn/80.4.936

Eroglu, S. (2018). "Chapter Four - Metal transport in the developing plant seed," in Advances in Botanical Research, ed. C. Maurel (Cambridge, MA: Academic Press), 91-113. doi: 10.1016/bs.abr.2018.09.010

Eroglu, S., Giehl, R. F. H., Meier, B., Takahashi, M., Terada, Y., Ignatyev, K., et al. (2017). Metal tolerance protein 8 mediates manganese homeostasis and iron reallocation during seed development and germination. Plant Physiol. 174, 1633-1647. doi: 10.1104/pp.16.01646

Eroglu, S., Meier, B., von Wirén, N., and Peiter, E. (2016). The vacuolar manganese transporter MTP8 determines tolerance to iron deficiency-induced chlorosis in Arabidopsis. Plant Physiol. 170, 1030-1045. doi: 10.1104/pp.15.01194

Finch-Savage, W. E., and Leubner-Metzger, G. (2006). Seed dormancy and the control of germination. New Phytol. 171, 501-523.

Finn, R. D., Coggill, P., Eberhardt, R. Y., Eddy, S. R., Mistry, J., Mitchell, A. L., et al. (2015). The Pfam protein families database: towards a more sustainable future. Nucleic Acids Res. 44, 279-285. doi: 10.1093/nar/gkv1344

Forbis, T. A., Floyd, S. K., and de Queiroz, A. (2002). The evolution of embryo size in angiosperms and other seed plants: implications for the evolution of seed dormancy. Evolution 56, 2112-2125. doi: 10.1111/j.0014-3820.2002.tb00137.x

Gasteiger, E., Hoogland, C., Gattiker, A., Wilkins, M. R., Appel, R. D., and Bairoch, A. (2005). "Protein identification and analysis tools on the ExPASy server," in The Proteomics Protocols Handbook, ed. J. M. Walker (Berlin: Springer), 571-607. doi: 10.1385/1-59259-890-0:571

Gibson, R. S., Bailey, K. B., Gibbs, M., and Ferguson, E. L. (2010). A review of phytate, iron, zinc, and calcium concentrations in plant-based complementary foods used in low-income countries and implications for bioavailability. Food Nutr. Bull. 31, 134-146.

Goodstein, D. M., Shu, S., Howson, R., Neupane, R., Hayes, R. D., Fazo, J., et al. (2011). Phytozome: a comparative platform for green plant genomics. Nucleic Acids Res. 40, 1178-1186. doi: 10.1093/nar/gkr944

Hedges, S. B., and Kumar, S. (2009). The Timetree of Life. Oxford: OUP Oxford.

Hegeman, C. E., and Grabau, E. A. (2001). A novel phytase with sequence similarity to purple acid phosphatases is expressed in cotyledons of germinating soybean seedlings. Plant Physiol. 126, 1598-1608. doi: 10.1104/pp.126.4.1598

Herman, E. M., and Larkins, B. A. (1999). Protein storage bodies and vacuoles. Plant Cell 11, 601-613. doi: 10.1105/tpc.11.4.601

Hurrell, R., and Egli, I. (2010). Iron bioavailability and dietary reference values. Am. J. Clin. Nutr. 91, 1461-1467.

Ibeas, M. A., Grant-Grant, S., Navarro, N., Perez, M. F., and Roschzttardtz, H. (2017). Dynamic subcellular localization of iron during embryo development in Brassicaceae seeds. Front. Plant Sci. 8:2186. doi: 10.3389/fpls.2017.02186

Iwai, T., Takahashi, M., Oda, K., Terada, Y., and Yoshida, K. T. (2012). Dynamic changes in the distribution of minerals in relation to phytic-acid accumulation during rice seed development. Plant Physiol. 160, 2007-2014. doi: 10.1104/pp. 112.206573

Karydas, A. G., Czyzycki, M., Leani, J. J., Migliori, A., Osan, J., Bogovac, M., et al. (2018). An IAEA multi-technique X-ray spectrometry endstation at Elettra Sincrotrone Trieste: benchmarking results and interdisciplinary applications. J. Synchrotron Radiat. 25, 189-203. doi: 10.1107/S1600577517016332
Kassebaum, N. J., Jasrasaria, R., Naghavi, M., Wulf, S. K., Johns, N., Lozano, R., et al. (2014). A systematic analysis of global anemia burden from 1990 to 2010. Blood 123, 615-624. doi: 10.1182/blood-2013-06-508325

Kim, S. A., Punshon, T., Lanzirotti, A., Li, L., Alonso, J. M., Ecker, J. R., et al. (2006). Localization of iron in Arabidopsis seed requires the vacuolar membrane transporter VIT1. Science 314, 1295-1298. doi: 10.1126/science.1132563

Kumar, S., Stecher, G., and Tamura, K. (2016). MEGA7: molecular evolutionary genetics analysis version 7.0 for bigger datasets. Mol. Biol. Evol. 33, 1870-1874. doi: $10.1093 / \mathrm{molbev} / \mathrm{msw} 054$

Kump, P., and Vogel-Mikuš, K. (2018). Quantification of 2D elemental distribution maps of intermediate-thick biological sections by low energy synchrotron \$lupmu\$-X-ray fluorescence spectrometry. J. Instrum. 13:C05014.

Lanquar, V., Lelièvre, F., Bolte, S., Hamès, C., Alcon, C., Neumann, D., et al. (2005). Mobilization of vacuolar iron by AtNRAMP3 and AtNRAMP4 is essential for seed germination on low iron. EMBO J. 24, 4041-4051. doi: 10.1038/sj.emboj. 7600864

Lee, S., Jeon, U. S., Lee, S. J., Kim, Y.-K., Persson, D. P., Husted, S., et al. (2009). Iron fortification of rice seeds through activation of the nicotianamine synthase gene. Proc. Natl. Acad. Sci. U.S.A. 106, 22014-22019. doi: 10.1073/pnas.0910950106

Letunic, I., and Bork, P. (2016). Interactive tree of life (iTOL) v3: an online tool for the display and annotation of phylogenetic and other trees. Nucleic Acids Res. 44, 242-245. doi: 10.1093/nar/gkw290

Lombardi-Boccia, G., De Santis, N., Di Lullo, G., and Carnovale, E. (1995). Impact of processing on Fe dialysability from bean (Phaseolus vulgaris L.). Food Chem. 2, 191-195. doi: 10.1016/0308-8146(95)90787-8

Lu, L., Tian, S., Liao, H., Zhang, J., Yang, X., Labavitch, J. M., et al. (2013). Analysis of metal element distributions in rice (Oryza sativa L.) seeds and relocation during germination based on X-Ray fluorescence imaging of $\mathrm{Zn}, \mathrm{Fe}, \mathrm{K}, \mathrm{Ca}$, and Mn. PLoS One 8:e57360. doi: 10.1371/journal.pone.0057360

Meguro, R., Asano, Y., Odagiri, S., Li, C., Iwatsuki, H., and Shoumura, K. (2007). Nonheme-iron histochemistry for light and electron microscopy: a historical, theoretical and technical review. Arch. Histol. Cytol. 70, 1-19. doi: 10.1679/ aohc.70.1

Moraghan, J., Padilla, J., Etchevers, J., Grafton, K., and Acosta-Gallegos, J. (2002). Iron accumulation in seed of common bean. Plant Soil 246, 175-183.

Murgia, I., Arosio, P., Tarantino, D., and Soave, C. (2012). Biofortification for combating 'hidden hunger' for iron. Trends Plant Sci. 17, 47-55. doi: 10.1016/ j.tplants.2011.10.003

Otegui, M. S., Capp, R., and Staehelin, L. A. (2002). Developing seeds of Arabidopsis store different minerals in two types of vacuoles and in the endoplasmic reticulum. Plant Cell 14, 1311-1327. doi: 10.1105/tpc.010486

Paciorek, T., Sauer, M., Balla, J., Wiśniewska, J., and Friml, J. (2006). Immunocytochemical technique for protein localization in sections of plant tissues. Nat. Protoc. 1, 104-107. doi: 10.1038/nprot.2006.16

Park, M.-J., Jung, H.-S., Kim, Y.-J., Kwon, Y.-J., Lee, J.-K., and Park, C.-M. (2014). High-sensitivity fluorescence imaging of iron in plant tissues. Chem. Commun. 50, 8547-8549. doi: $10.1039 / \mathrm{c} 4 \mathrm{cc} 02132 \mathrm{k}$

Ramos, M. S., Khodja, H., Mary, V., and Thomine, S. (2013). Using $\mu$ PIXE for quantitative mapping of metal concentration in Arabidopsis thaliana seeds. Front. Plant Sci. 4:168. doi: 10.3389/fpls.2013.00168

Reddy, C. S., Kim, S.-C., and Kaul, T. (2017). Genetically modified phytase crops role in sustainable plant and animal nutrition and ecological development: a review. 3 Biotech 7:195. doi: 10.1007/s13205-017-0797-3

Regvar, M., Eichert, D., Kaulich, B., Gianoncelli, A., Pongrac, P., Vogel-Mikuš, K., et al. (2011). New insights into globoids of protein storage vacuoles in wheat aleurone using synchrotron soft X-ray microscopy. J. Exp. Bot. 62, 3929-3939. doi: $10.1093 /$ jxb/err090

Roschzttardtz, H., Conejero, G., Curie, C., and Mari, S. (2009). Identification of the endodermal vacuole as the iron storage compartment in the Arabidopsis embryo. Plant Physiol. 151, 1329-1338. doi: 10.1104/pp.109.144444

Santos-Buelga, C., and Scalbert, A. (2000). Proanthocyanidins and tannin-like compounds - nature, occurrence, dietary intake and effects on nutrition and health. J. Sci. Food Agric. 80, 1094-1117. doi: 10.1002/(sici)10970010(20000515)80:7<1094::aid-jsfa569>3.0.co;2-1

Shahzad, Z., Rouached, H., and Rakha, A. (2014). Combating mineral malnutrition through iron and zinc biofortification of cereals: Fe and $\mathrm{Zn}$ biofortification of cereals.... Compr. Rev. Food Sci. Food Saf. 13, 329-346. doi: 10.1111/15414337.12063 
Shi, J., Wang, H., Schellin, K., Li, B., Faller, M., Stoop, J. M., et al. (2007). Embryospecific silencing of a transporter reduces phytic acid content of maize and soybean seeds. Nat. Biotechnol. 25, 930-937. doi: 10.1038/nbt1322

Singh, S. P., Vogel-Mikuš, K., Arèon, I., Vavpetiè, P., Jeromel, L., Pelicon, P., et al. (2013). Pattern of iron distribution in maternal and filial tissues in wheat grains with contrasting levels of iron. J. Exp. Bot. 64, 3249-3260. doi: 10.1093/jxb/ ert 160

Singh, S. P., Vogel-Mikuš, K., Vavpetiè, P., Jeromel, L., Pelicon, P., Kumar, J., et al. (2014). Spatial X-ray fluorescence micro-imaging of minerals in grain tissues of wheat and related genotypes. Planta 240, 277-289. doi: 10.1007/s00425-0142084-4

Solé, V. A. A., Papillon, E., Cotte, M., Walter, P. H., and Susini, J. (2007). A multiplatform code for the analysis of energy-dispersive X-ray fluorescence spectra. Spectrochim. Acta Part B At. Spectrosc. 62, 63-68. doi: 10.1016/j.sab. 2006.12.002

Soltis, P. S. (2005). Ancient and recent polyploidy in angiosperms. New Phytol. 166, 5-8. doi: 10.1111/j.1469-8137.2005.01379.x

Stacey, M. G., Patel, A., McClain, W. E., Mathieu, M., Remley, M., Rogers, E. E., et al. (2008). The Arabidopsis AtOPT3 protein functions in metal homeostasis and movement of iron to developing seeds. Plant Physiol. 146, 589-601. doi: 10.1104/pp.107.108183

Tusnady, G. E., and Simon, I. (2001). The HMMTOP transmembrane topology prediction server. Bioinformatics 17, 849-850. doi: 10.1093/bioinformatics/17. 9.849

Vatansever, R., Filiz, E., and Eroglu, S. (2017). Genome-wide exploration of metal tolerance protein (MTP) genes in common wheat (Triticum aestivum): insights into metal homeostasis and biofortification. Biometals 30, 217-235. doi: 10.1007/s10534-017-9997-x

Vogel-Mikus, K., Drobne, D., and Regvar, M. (2005). Zn, Cd and Pb accumulation and arbuscular mycorrhizal colonisation of pennycress Thlaspi praecox Wulf. (Brassicaceae) from the vicinity of a lead mine and smelter in Slovenia. Environ. Pollut. Barking Essex 1987, 233-242. doi: 10.1016/j.envpol.2004.06.021

Vogel-Mikus, K., Pongrac, P., Kump, P., Necemer, M., Simcic, J., Pelicon, P., et al. (2007). Localisation and quantification of elements within seeds of $\mathrm{Cd} / \mathrm{Zn}$ hyperaccumulator Thlaspi praecox by micro-PIXE. Environ. Pollut. Barking Essex 1987, 50-59. doi: 10.1016/j.envpol.2006.08.026
Vogel-Mikuš, K., Pongrac, P., and Pelicon, P. (2014). MicroPIXE elemental mapping for ionome studies of crop plants. Int. J. PIXE 24, 217-233. doi: 10.1142/s0129083514 400142

Vogel-Mikuš, K., Simčič, J., Pelicon, P., Budnar, M., Kump, P., Neèemer, M., et al. (2008). Comparison of essential and non-essential element distribution in leaves of the $\mathrm{Cd} / \mathrm{Zn}$ hyperaccumulator Thlaspi praecox as revealed by micro-PIXE. Plant Cell Environ. 31, 1484-1496. doi: 10.1111/j.1365-3040.2008. 01858.x

Waldo, G. S., Wright, E., Whang, Z. H., Briat, J. F., Theil, E. C., and Sayers, D. E. (1995). Formation of the ferritin iron mineral occurs in plastids (An X-Ray Absorption Spectroscopy Study. Plant Physiol. 109, 797-802. doi: 10.1104/pp. 109.3.797

Warkentin, T. D., Delgerjav, O., Arganosa, G., Rehman, A. U., Bett, K. E., Anbessa, Y., et al. (2012). Development and characterization of low-phytate pea. Crop Sci. 52, 74-78.

Zhang, Y., Xu, Y.-H., Yi, H.-Y., and Gong, J.-M. (2012). Vacuolar membrane transporters OsVIT1 and OsVIT2 modulate iron translocation between flag leaves and seeds in rice. Plant J. 72, 400-410. doi: 10.1111/j.1365-313X.2012. 05088.x

Zuckerkandl, E., and Pauling, L. (1965). "Evolutionary divergence and convergence in proteins," in Evolving Genes and Proteins, eds V. Bryson and H. J. Vogel (New York, NY: Academic Press), 97-166. doi: 10.1016/b978-1-4832-2734-4. 50017-6

Conflict of Interest Statement: The authors declare that the research was conducted in the absence of any commercial or financial relationships that could be construed as a potential conflict of interest.

Copyright (C) 2019 Eroglu, Karaca, Vogel-Mikus, Kavčič, Filiz and Tanyolac. This is an open-access article distributed under the terms of the Creative Commons Attribution License (CC BY). The use, distribution or reproduction in other forums is permitted, provided the original author(s) and the copyright owner(s) are credited and that the original publication in this journal is cited, in accordance with accepted academic practice. No use, distribution or reproduction is permitted which does not comply with these terms. 